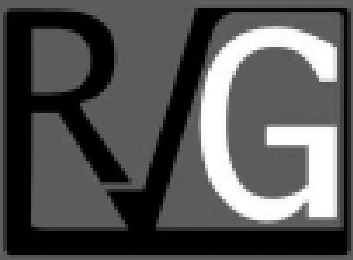

Año 24 No. 88

Octubre - Diciembre 2019

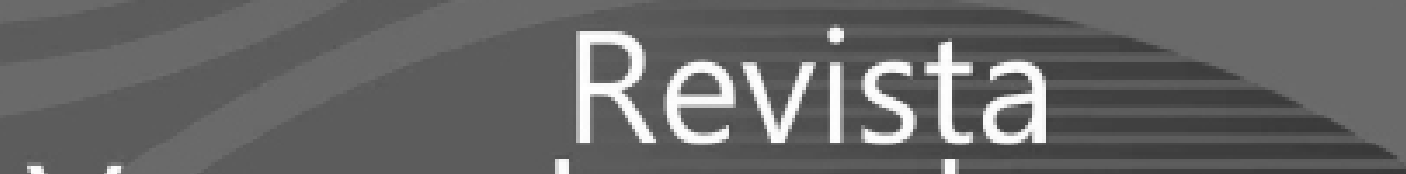

Venezolana de

verencla

UNIVERSIDAD DEL ZULIA (LUZ)

Facultad de Ciencias Económicas y Sociales

Centro de Estudios de la Empresa 


\title{
Contabilidad gubernamental y normas internacionales de contabilidad para el sector público ecuatoriano ${ }^{1}$
}

\author{
Reyes Reinoso, Johanna Rosalí ${ }^{2}$ \\ Reyes Cárdenas, Narciza ${ }^{3}$ \\ Cárdenas Muñoz, Jorge ${ }^{4}$
}

\section{Resumen}

Las Normas Internacionales de Contabilidad para el Sector Público (NICSP), establecen los requisitos para la elaboración de informes financieros que emiten las entidades del sector público no financiero, las cuales son de obligatorio cumplimiento por parte de los Estados. En el caso de Ecuador estas normas entran en vigencia a partir de enero del 2020. El artículo tiene como objetivo analizar la convergencia de la contabilidad gubernamental de los Gobiernos Autónomos Descentralizados Municipales del Ecuador con las Normas Internacionales de Contabilidad para el Sector Público, concretamente en la Provincia del Cañar. El estudio se realizó entre enero y julio del 2019 en el cual se aplicó una encuesta con el fin de conocer la opinión de los funcionarios sobre los lineamientos del ente rector en relación al proceso y cronograma de implementación de las NICSP. Los resultados muestran los problemas que confrontan los gobiernos autónomos de la provincia el Cañar para adaptarse a los requerimientos de la convergencia a la normativa internacional lo que origina severas dificultades para su implementación.

Palabras clave: Contabilidad gubernamental; Normas Internacionales de Contabilidad Sector Publico (NICSP); Gobiernos Autónomos Descentralizados (GADS).

Recibido: 27-06-19 Aceptado: 25-09-19

1 Esta investigación deriva de la Unidad Académica de Administración de la Universidad Católica de Cuenca, se inserta en la línea de investigación Gestión empresarial con responsabilidad social, ámbito área Contable buscando responder a las necesidades del Sector Público no Financiero del Ecuador.

2 Magister en Auditoría Integral por la Universidad Técnica Particular de Loja. Docente Investigador de la Universidad Católica de Cuenca, adscrita a la Unidad Académica de Administración. ORCID: 0000-0001-8663-4677. jreyesr@ ucacue.edu.ec

3 Magister en Contabilidad y Finanzas por la Universidad del Azuay. Docente de la Universidad Católica de Cuenca, adscrita a la Unidad Académica de Administración. ORCID: 000003-4230-1541. nareyesc@ucacue.edu.ec

$4 \quad$ Magister en Auditoría Integral por la Universidad Técnica Particular de Loja. Docente de la Universidad Católica de Cuenca, adscrita a la Unidad Académica de Administración. ORCID: 0000-0001-8663-4677. jvcardenasm@ ucacue.edu.ec 


\title{
Government accounting and international accounting standards for the Ecuadorian public sector
}

\begin{abstract}
The International Accounting Standards for the Public Sector (IPSAS), establish the requirements for the preparation of financial reports issued by non-financial public sector entities, which are mandatory for States to comply with. In the case of Ecuador, these regulations are effective as of January 2020. The article aims to analyze the convergence of government accounting of the Autonomous Municipal Decentralized Governments of Ecuador with the International Accounting Standards for the Public Sector, specifically in the Province of Cañar. The study was carried out between January and July of 2019 in which a survey was applied in order to know the opinion of the officials on the guidelines of the governing body in relation to the process and implementation schedule of IPSAS. The results show the problems faced by the autonomous governments of the Cañar province to adapt to the requirements of convergence to international regulations, which causes severe difficulties for its implementation
\end{abstract}

Keywords: Government Accounting; International; Public Sector Accounting Standards (IPSAS); Decentralized Autonomous Governments (GADS).

\section{Introducción}

La Contabilidad Gubernamental constituye una herramienta financiera que permite la cuantificación de la información financiera del sector público, además facilita el reconocimiento del patrimonio público, la gestión y evaluación de las decisiones en relación a la producción de bienes y servicios en beneficios de sus ciudadanos.

En Ecuador, el Código Orgánico de Planificación y Finanzas Públicas (COPYFP), lo define como el proceso de registro sistemático, cronológico y secuencial de las operaciones patrimoniales y presupuestarias de las entidades y organismos del Sector Público no Financiero, expresadas en términos monetarios, desde la entrada original a los registros contables hasta la presentación de los estados financieros; la centralización, consolidación y la interpretación de la información; comprende además los principios, normas, métodos y procedimientos correspondientes a la materia. (Asamblea Nacional, 2010).

Ahora bien, en los últimos años se ha venido produciendo una serie de cambios en los que se desenvuelve el sector público que ha impactado con mayor énfasis la interrelación entre los gobiernos y sus ciudadanos, fundamentalmente aquellos vinculados a la visión de la gestión pública y el carácter estratégico que tiene los sistemas de contabilidad a la hora de 
evaluar la gestión gubernamental.

En este contexto, a nivel internacional se adelanta una serie de discusiones y elaboración de modelos contables con la finalidad de asegurar la calidad y transparencia de la información contable pública, para lo cual se propuso las Normas Internacionales de Contabilidad del Sector Público (NICSP) conocidas también por sus siglas en inglés como IPSAS (International Public Sector Accounting Standards.

Estas normas, según Ablan (2013: 224), establecen "los requisitos para el reconocimiento, medición, presentación y revelación de transacciones y hechos en los estados financieros con propósito general". En este sentido, las NICSP presentan las prácticas internacionales de información financiera para su aplicación a entidades públicas, específicamente en los estados financieros.

Por otro lado, Ecuador forma parte del acuerdo Latinoamericano y del Caribe para la implementación de las NICSP, el cual definió un cronograma para su implementación. Países como Chile y Colombia implementaron estas normas en el año 2018 y en Perú en el año 2019. En tanto, para Ecuador su obligatoriedad es a partir de enero del 2020.

Para llevar a cabo dicha implementación el Reglamento del COPYFP señala "en la formulación de la normativa contable gubernamental el Ministerio de Finanzas considerará las Normas Internacionales de Contabilidad para el sector público y será de cumplimiento obligatorio para las entidades del Presupuesto General del Estado y gobiernos autónomos descentralizados" (Presidencia de la República, 2014).

En este sentido, a partir de las exigencias establecidas por el Ministerio de Finanzas, los Gobiernos Autónomos Descentralizados Municipales (GADS) deben manejar de manera exhaustiva los lineamientos y procesos esenciales para poder converger de forma óptima con la normativa a ser implementada en el año 2020.

Las consecuencias derivadas de la no implementación y el manejo inadecuado de las NICSP, afectará la estandarización de la información financiera que deben generar, pues, la contabilidad gubernamental tiene como fines la comparabilidad, la gestión y fundamentalmente el control de los recursos públicos, previniendo fraudes, sobornos y funcionamientos inapropiados que hablarán de la eficiencia o no de la administración pública-gubernamental en las realidades latinoamericanas, marcadas por exigencias que dan la implementación de estas normas.

Por ello, es necesario establecer estrategias que permitan a las entidades públicas concluir con el proceso conforme a lo planificado en el instructivo emitido por el ente rector del país, es decir, el Ministerio de Finanzas, dando cumplimiento al cronograma de implementación de las NICSP en América Latina.

En consecuencia, el presente trabajo consiste en analizar el grado de avance de la convergencia de la contabilidad gubernamental de los Gobiernos Autónomos Descentralizados Municipales del Ecuador con las Normas Internacionales de Contabilidad para el Sector Público (NICSP), concretamente en la Provincia del Cañar en el periodo enero - julio del 2019. Se trata de una investigación con enfoque cuantitativo, partiendo de un nivel exploratorio descriptivo a través de un diseño no experimental, cuya unidad de 
Reyes Reinoso, Johanna Rosalí; Reyes Cárdenas, Narciza y Cárdenas Muñoz, Jorge_

análisis son los gobiernos autónomos descentralizados.

Para la recolección de los datos se aplicó una encuesta a los altos funcionarios de los cinco (5) gobiernos autónomos descentralizados de la provincia del Cañar en relación al proceso y cronograma de implementación de las NICSP. Estos gobiernos autónomos son: La Troncal, Suscal, Déleg, Biblián y Azogues (capital de la provincia), cantones que por su monto presupuestario se clasifican en pequeños y mediano.

Para ello, se diseñó un cuestionario de preguntas cerradas concernientes al instructivo emitido por el Ministerio de Finanzas para la convergencia de la Normativa de Contabilidad Gubernamental a las NICSP a partir del 1 de enero de 2020, que deben tener las entidades públicas no financieras del Ecuador. Dicho instrumento está constituido por 17 ítems para respuestas dicotómicas con puntuación cuantitativa, relacionadas con el conocimiento de las normas y depuración de saldos contables.

La información levantada mediante el instrumento fue tabulada en una tabla matriz de frecuencia absoluta y relaciones porcentuales, lo que permitió visualizar con facilidad los resultados para su análisis a través de la estadística descriptiva, apoyados con el software Statistical Package for the Social Sciences (SPSS versión 22).

\section{Devenir histórico de la contabilidad. Breve reseña histórica}

La contabilidad tiene sus orígenes en la historia cuando el hombre tuvo necesidad de contar y anotar, tal actividad, según Casal (2007:20), fue evolucionado "conforme se perfeccionó la propiedad y el comercio". En este sentido, el desarrollo de la contabilidad se presenta a la par de la sociedad y su extenso camino, pasado por diferentes etapas históricas. Montesino (1993), considera cuatro periodos en el desarrollo de la contabilidad, tal como se observa en la figura 1.

Sus inicios, se pueden ubicar en el periodo empírico, en la civilización de Mesopotamia, la cual asume una forma de escritura contable del cálculo abstracto. Esta práctica de escritura contable pasó de ser personal y familiar, a empresarial y estatal, cuya característica principal fue basarse en un libro memorial.

Un segundo periodo, el que se le conoce, como el periodo clásico, ubicándolo hasta la primera guerra mundial, en el que prevalece el desarrollo de la partida simple que luego se trasformaría en el sistema de registro de mayor aceptación.

Con la aparición del capitalismo y el desarrollo de las actividades mercantiles y del comercio, se introduce el principio de racionalidad en las operaciones, es decir, el saber qué ocurrió, para ello es necesario un sistema de registro más completo que permita al empresario contar con información sobre los acontecimientos económicos de su empresa, dado lugar a la aparición de la partida doble, como consecuencia de un complejo proceso social y económico en el que se busca un control racional de la riqueza.

Con el surgimiento de la imprenta se beneficia la escritura contable, con la cual se expende el conocimiento de los principios contables, entre los se cuenta con los aportes del matemático Pietra, 


\section{Figura 1 \\ Periodos históricos de la contabilidad}

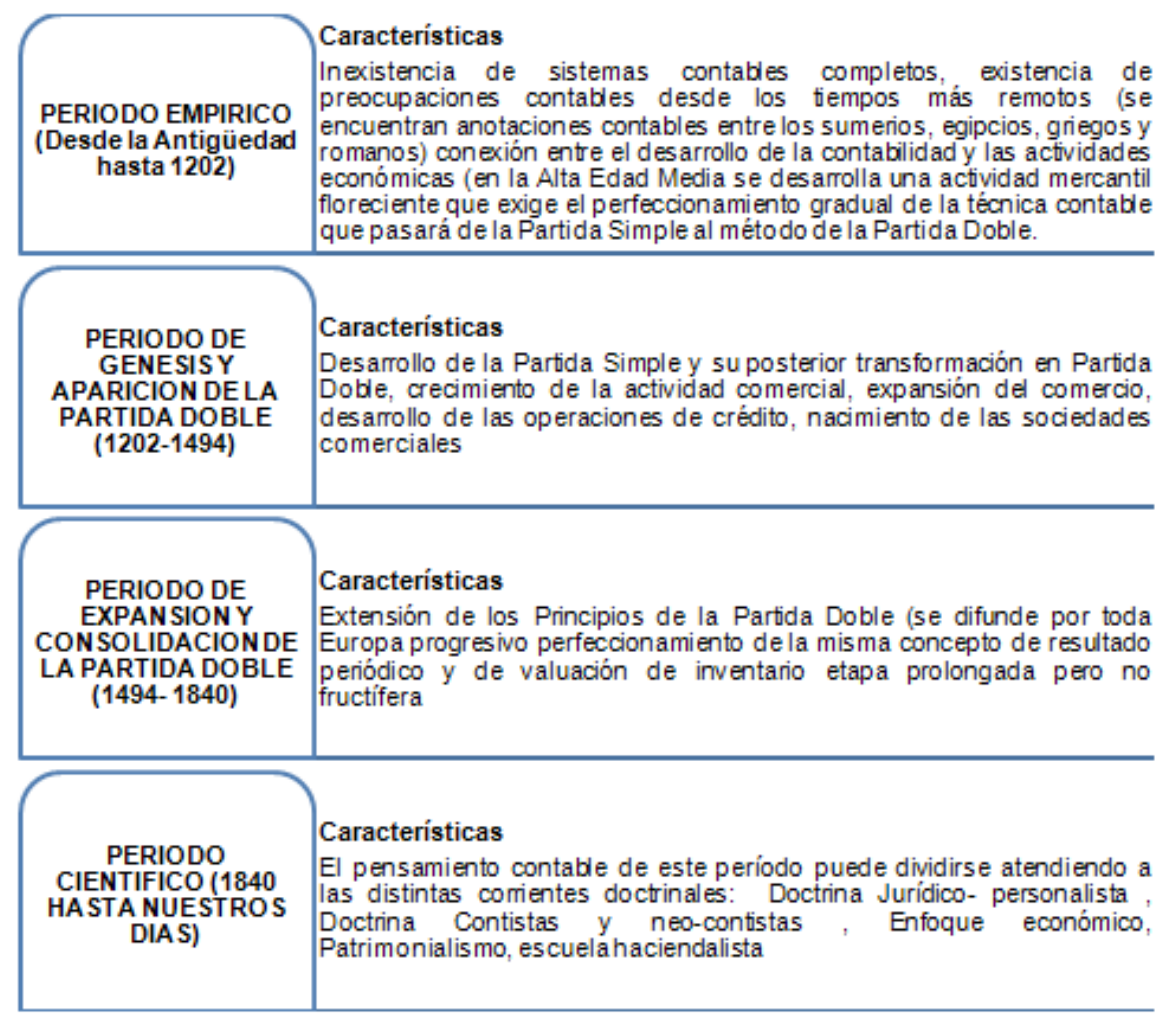

Fuente: Montesinos (1993).

que evidencia la correlación de débitos y crédito con la naturaleza de los hechos patrimoniales (Ramos, 2012).

En tanto en Inglaterra, Dodson describe el procedimiento de cálculos de los costos por lotes o pedidos. Los esfuerzos por consolidar los procedimientos contables, también tienen lugar en Francia, en los que se introduce la clasificación racional de los hechos patrimoniales a través de la división de entendimiento entre la personalidad de la empresa y su propietario (López, 2002).

La forma de entender el objeto de estudio y la metodología de la contabilidad florecen con las doctrinas del contismo, personalismo, controlismo, haciendalismo y patrimonialismo, que representaron los horizontes del arte de registrar, iniciándose así, el periodo científico hasta la actualidad. 
Reyes Reinoso, Johanna Rosalí; Reyes Cárdenas, Narciza y Cárdenas Muñoz, Jorge_

La doctrina contista tiene como objeto de estudio de la contabilidad la cuenta, en la que explica el funcionamiento de la partida doble partiendo del principio básico de que "quien recibe un valor es siempre deudor del mismo, y quien lo entrega es acreedor" (Ramos, 2012:42).

La doctrina jurídico- personalista vincula la contabilidad con el derecho, con la posesión. Para esta escuela, los fenómenos contables se relacionan con lo jurídico adminsitrativo frente al patrimonio.

Con la corriente haciendalista se expone el concepto de hacienda como un conjunto de personas y medios materiales e inmateriales que conducen a cumplir una finalidad y expone que dentro del estudio de la contabilidad se debe considerar los fenómenos de la administración económica de la hacienda.

Por otro lado, la corriente contable patrimonialista considera a la contabilidad como la ciencia que estudia los fenómenos del patrimonio haciendal, en cuanto "a su estado estático, dinámico y de revelación” (Ramos, 2012:46). Refiriéndose claramente a los estados financieros.

López (2002:23), plantea que la estatica patrimonial da cuenta de la posición financiera en un momento determinado, la dinámica patrimonial a los resultados de la empresa en torno a su gestión, y las revelaciones corresponden a las explicaciones de los primeros.

En suma, el desarrollo histórico de la contabilidad, la ha configurado como ciencia, en la que subsisten esfuerzos que se manifiestan en sus núcleos epistemológicos y metodológicos. En este sentido, esta investigación entiende que en el fenómeno contable subyace una cosmovisión desde donde se aborda el problema de la cientificidad de la contabilidad y su naturaleza social. En esta perspectiva, es propio de la teoría contable explicar y predecir la práctica contable, entendida en un sentido amplio, para solucionar problemáticas propia de su naturaleza o conflictos de fronteras con otras disciplinas. Es por ello, que a comienzos del siglo XXI el ritmo de la globalización trae consigo nuevos enfoque y paradigmas, en tanto, la contabilidad como ciencia tiende a transformase para responder a estos rápidos y profundos cambios.

\section{Contabilidad gubernamental}

La contabilidad gubernamental en la actualidad se considera como un instrumento indispensable en el sector público, pues permite observar los registros económicos, de acuerdo a las bases legales, de las transacciones efectuadas por las entidades públicas. Su objetivo es proporcionar información destinada al control y evaluación de la eficacia de los entes públicos.

Además, se considera como la disciplina que trata el estudio económico y financiero de la hacienda pública, la cual permite evaluar la gestión administrativa del Estado. Según Alvarado (1995:36), es un conjunto de principios y normas que comprenden niveles operacionales como áreas contables, documentos fuentes, informes de movimiento por áreas, plan de cuentas, libros principales y registros auxiliares de contabilidad, estados financieros y presupuestarios, archivos de las transacciones, así como los procedimientos de registración y de elaboración e interpretación de los estados contables.

Por tanto, constituye un sistema de información sobre el proceso financiero 
de las organizaciones públicas, que operan en función de la teoría contable, para su estudio, diseño y operación con la finalidad de ejercer el control real y efectivo sobre el patrimonio público.

Es así, como el sistema contable público suministra información sobre créditos, destino del gasto y control interno para garantizar la coordinación y estabilidad macroeconómica en los distintos niveles del gasto gubernamental. Para Lara, Toledo, \& Gómez (2012), la contabilidad gubernamental permite cuantificar la cantidad de recursos que utilizarán los funcionarios públicos en la producción de bienes y en la generación de servicios.

Por tanto, genera información útil que permite mejorar la toma de decisiones en la gerencia pública, ya que cuenta

... con características relevantes, representación fiel, comparable, verificable, oportuna y comprensible, lo cual permite determinar los resultados macroeconómicos en los estados de cuenta, el producto bruto, el ingreso nacional disponible, la acumulación y financiamiento del capital y las transacciones externas, así como la solvencia o no de los entes descentralizados(Mejías, 2011:29).

La contabilidad gubernamental realiza el análisis de las operaciones financieras del estado, basándose en los principios de la contabilidad para mantener la credibilidad en la administración de los ingresos, gastos e inversiones públicas, control y efectividad del uso de los recursos, presentando una información contable ordenada con los documentos que sustente el hecho económico de tal forma que facilite las tareas de control y auditoría interna y/o externa.
El sistema de contabilidad gubernamental debe estar estructurado sobre la base de una descentralización de los registros y de los datos para fines de agregación y consolidación, de acuerdo con la estructura organizacional del Estado, es decir, se estructura como un sistema integral, único y uniforme.

La característica de este sistema es establecer un proceso de integración ordenado con controles en diferentes áreas contables para producir información financiera oportuna y confiable. Este proceso se inicia con la recepción de la información, análisis de la documentación, registro de hechos económicos y por último la elaboración de los estados financieros que revelan la administración o uso que se les ha dado a los recursos públicos.

En el caso de Ecuador, los principios de la contabilidad gubernamental constituyen las bases o los fundamentos que rigen la materia contable en su aplicación gubernamental y como tales deben ser estrictamente observados. La observancia de estos principios durante el proceso contable hace que la información que se genere sea confiable y comprensible. Estos principios son: medición económica, igualdad contable, costos históricos, devengado, realización, reexpresión contable y consolidación. Además, según lo establece la Ley Orgánica de Administración Financiera, el Ministerio de Economía y Finanzas es el órgano rector del Sistema Nacional de Presupuesto Público y tiene la obligación de elaborar el sistema de información que registre los resultados que se deriven de las operaciones presupuestarias, de forma compatible con el sistema de Contabilidad Gubernamental. 
Reyes Reinoso, Johanna Rosalí; Reyes Cárdenas, Narciza y Cárdenas Muñoz, Jorge_

\subsection{Normativa Internacional de Contabilidad del Sector Público (NICSP)}

Con el fin de garantizar la calidad y transparencia de los estados financieros en el Sector Público a nivel internacional se propuso la adopción de las Normas internacionales de Contabilidad del Sector Público (NICSP), reestructurando las diferentes normas de aplicación en los sistemas de Contabilidad Gubernamental basadas en la cultura política y socioeconómica de cada país. Es la Federación Internacional de Contadores (IFAC, por sus siglas en inglés), a través de la junta técnica denominada Consejo de Normas Internacionales de Contabilidad para el Sector Público (IPSASB por sus siglas en inglés), quien emite estas normas.

Esta iniciativa surge debido a la falta de uniformidad de criterio y baja calidad de la información que contiene los informes financieros emitidos ante terceros por los responsables de la gerencia pública. En tal sentido, las NICSP establecen los requisitos para la elaboración de informes financieros que emiten los gobiernos y otras entidades del sector público que no sean empresas comerciales del gobierno.

Las NICSP sobre el método contable de acumulación, se basan en las Normas Internacionales de Información Financiera (NIIF) que son emitidas por el Consejo de Normas Internacionales de Contabilidad (International Accounting Standards Board/IASB) en la medida en que los requisitos incluidos en esas normas se apliquen al sector público.

Rodríguez, Navarro, \& Alcaraz, las definen como un conjunto de normas o leyes que establecen la información que deben presentarse en los estados financieros del sector público no financiero y la forma en que esa información debe aparecer, en dichos estados (2014:53). Las NICSP no son leyes físicas o naturales que esperaban su descubrimiento, sino más bien normas que el hombre, de acuerdo con sus experiencias comerciales $y$ financieras, sigue creyendo que esto es verdad y ha considerado de importancia en la presentación de la información financiera.

Son normas contables gubernamentales de alta calidad, cuyo objetivo es reflejar la esencia económica de las operaciones de las entidades públicas, y presentar una imagen fiel de la situación financiera de dichas entidades. Estas normas pretenden contar con la seguridad de la información financiera, económica, patrimonial e incluso presupuestaria fidedigna, digna de fe, fiable.

En tanto, Zerpa, Rosales \& Morales (2017), consideran que existen dos instituciones internacionales que regulan la contabilidad gubernamental como lo son: la Federación Internacional de Contadores, encargada de emitir lineamientos de contabilidad para el sector público y el Fondo Monetario Internacional, que busca sistemas integrados de administración de recursos financieros.

Para Gianchino (2011), los beneficios para los usuarios de los estados financieros del sector público respecto a las NICSP son: a) establecimientos de prácticas de información financiera adecuadas y b) la consistencia en la aplicación de dichas prácticas (tanto interna como entre países.

Por su parte Fuentes apuntala, que "disponer de una información comparable a nivel internacional beneficia a 
organismos supranacionales y a potenciales inversores internacionales interesados en el sector público, quienes requieren información comparable, además de servir de marco de referencia común para aquellos países que comienzan a modernizar su contabilidad pública" (2007:70)

En este sentido, varios países de América Latina han recibido apoyo financiero y técnico de diferentes organismos internacionales, a través de diversos programas que pretenden colaborar en el desarrollo económico de los mismos. Pero uno de los requisitos que imponen estos organismos para financiar proyectos gubernamentales es rendir cuenta de forma precisa y oportuna, por medio de unas normas mínimas en cuanto al sistema de contabilidad que los países beneficiados deban cumplir.

Por otra parte, las entidades públicas preparan estados financieros de tipo general para usuarios que no están en capacidad de acceder a información financiera, como por ejemplo, los ciudadanos que la requieren para evaluar el desempeño de sus gobernantes. Igualmente estas entidades preparan estados financieros específicos para cubrir necesidades para ciertos sectores del gobierno que requieren información especializada. La preparación de estos estados financieros se realiza por el método contable del devengado, que permite la elaboración del Estado en la Situación Financiera, el Estado de Resultados Financieros, el Estado de Flujos de Efectivos y el Estado de Cambios en el Patrimonio Neto. La utilización de las NICSP es más flexible que las NIC, porque no se impone su adopción. En el caso de que existan en el país regulaciones que rijan las prácticas contables y la presentación de los estados financieros, las NICSP no se superponen a estos criterios, sino que promueven que haya una armonización entre estas y las regulaciones internas de cada país para contribuir a una mayor comparabilidad.

\subsection{Normativa Internacional de Contabilidad del Sector Público en América Latina}

Los procesos de armonización contable gubernamental en América Latina, se caracterizan por una transición del ordenamiento jurídico a las Normas Internacionales de Contabilidad para el Sector Público, fundamentados en el principio del devengo, es decir pasar de un registro de hechos económicos basados en el flujo del efectivo al registro, cuando se genera el acto económico, cuando se adquiere el derecho o se contrae la obligación indiferentemente a que haya el egreso o ingreso de efectivo.

Esto conlleva a contar con una herramienta de gestión valiosa que fomente el control de los recursos públicos, la comparabilidad de la información financiera, la rendición de cuentas transparente, y a generar indicadores para la toma de decisiones, todo ello en normas estandarizadas en el mundo. En este sentido, diversos organismos internacionales promueven e impulsan este proceso de armonización, tal es el caso del Fondo Monetario Internacional (FMI), la Organización Mundial de Comercio (OMC), la Organización para la Cooperación y el Desarrollo Económico (OCDE) y el Banco Interamericano de Desarrollo (BID).

Este último, hace un seguimiento al proceso de convergencia hacia la adopción de las NICS en América Latina 
Reyes Reinoso, Johanna Rosalí; Reyes Cárdenas, Narciza y Cárdenas Muñoz, Jorge_

y El Caribe e indica que, la convergencia gradual de las normas nacionales hacia las NICSP de devengo, abre la puerta a una personalización y adaptación de los requerimientos de las NICSP, según las circunstancias específicas de cada país, a tal punto que no puedan cumplir con las normas, ya que uno de los objetivos principales es lograr una mejor comparabilidad de los datos, tanto a nivel de país como a nivel regional. Consecuentemente, los diferentes ajustes de las normas durante su adopción podrían debilitar este objetivo (BID 2017).

En tal sentido, los procesos y cronogramas individuales de conversión, al igual que los enfoques de reforma gradual y el nivel de alineación de los sistemas contables nacionales ofrece una foto de los procesos dinámicos de reforma que se realizan permanentemente en los países. Mientras algunos países todavía operan con un sistema de base de efectivo modificado y están actualmente en la etapa de preparación para la implementación de las NICSP de devengo, algunos países pioneros como Brasil, Chile, Colombia y Perú ya han logrado un estado avanzado de implementación de las NICSP (BID 2017).

Así mismo, la velocidad en el proceso de implementación de tales reformas se debe al hecho de que los gobiernos sienten presión interna y externa de sus jurisdicciones. La adopción de estas normas no solo mejora la comparabilidad de los datos, sino que aumenta la confianza entre los interesados. Por ejemplo, la aplicación de las NICSP o normas contables parecidas a las NICSP es un factor, que se consideran en las evaluaciones de riesgo fiduciario y otros índices, tales como los indicadores de desempeño de la gestión de las Finanzas Públicas (PEFA, por sus siglas en inglés) y los Informes del FMI sobre la Observación de Normas y Códigos (Reports on the Observance of Standards and Codes (ROSC).

Por consiguiente, la adopción de las NICSP normalmente tiene un impacto sobre la reputación de un país entre sus posibles inversionistas. (BID 2017)

Este organismo muestra el cronograma para la convergencia de los países latinoamericanos, tabla 1 :

\section{Tabla 1 \\ Cronograma de conversión de los países latinoamericanos

\begin{tabular}{lc}
\hline \multicolumn{1}{c}{ PAÍSES } & AÑO \\
\hline Chile y Colombia & 2018 \\
Perú & 2019 \\
Ecuador y El Salvador & 2020 \\
Honduras y República Dominicana & 2021 \\
Costa Rica y Guatemala & 2022 \\
Brasil & 2024 \\
Nicaragua, Panamá, Paraguay y Uruguay & No determinado \\
\hline \multicolumn{2}{l}{ Fuente: (Banco Interamericado de Desarrollo, 2017) }
\end{tabular}


Al comparar los sistemas contables actuales de los países con los requerimientos de las NICSP, se observa una amplia gama de niveles globales de alineación que varían entre el $11 \%$ y el $84 \%$. Mientras algunos países todavía operan con un sistema de base de efectivo modificado, algunos ya han logrado un estado avanzado de implementación de las NICSP y actualmente están en el período de transición provisto por la disposición transitoria de la NICSP individual o por la NICSP 33. (BID 2017)
En el análisis que realiza el Banco se muestra el nivel global de alineación a las NICPS, (gráfico 1), en donde se aprecia que Nueva Zelanda es el referente que ha avanzado un $99 \%$, Colombia un $84 \%$, Perú $82 \%$, Chile $67 \%$, y países en donde el avance ha sido inferior como Paraguay $11 \%$, Uruguay $13 \%$ y Nicaragua $17 \%$, de lo que se deduce que el proceso está en marcha sin embargo el tener a los países latinoamericanos en total convergencia va a tomar un tiempo considerable.

\section{Gráfico 1 \\ Porcentaje de niveles globales de alineación a las NICPS}

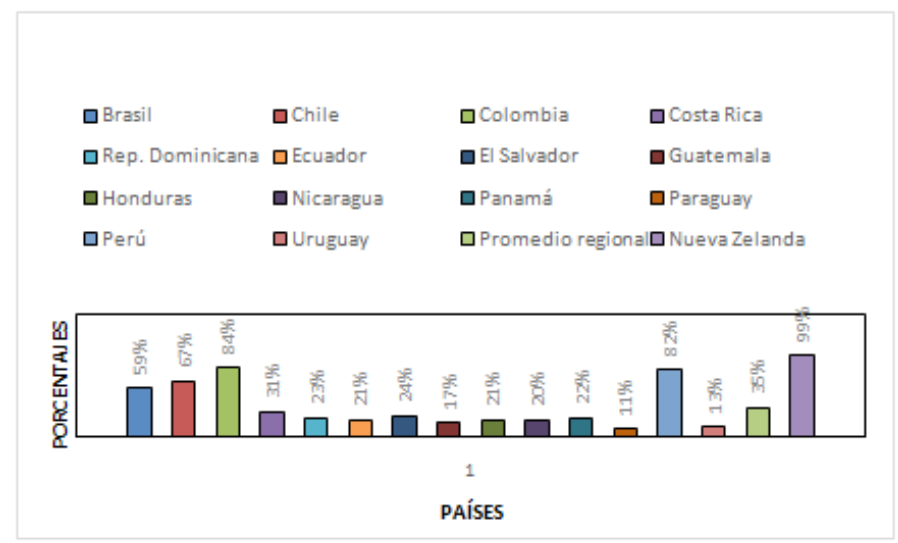

Fuente: (Banco Interamericado de Desarrollo, 2017)

\section{Normativa Internacional de Contabilidad para el Sector Público ecuatoriano}

La Normativa Internacional se fundamenta principalmente en el principio del devengo como reemplazo al del flujo del efectivo, que permite contar con información razonable, comparable, para gestión, pero fundamentalmente para el control de los recursos públicos que le permite rendir cuentas y que la población perciba la veracidad de la misma.

En Ecuador el 28 de diciembre de 2005, el Ministerio de Economía y Finanzas emite el Acuerdo Nro. 320, en el que consta el Manual de Contabilidad 
Gubernamental, dentro del cual contempla los principios contables al del devengado, lo que demuestra que ya se incursiona en la temática de la normativa internacional.

En octubre 19 de 2010, (Asamblea Nacional, 2010), se crea el Código Orgánico de Planificación y Finanzas Públicas (COPYFP) en el cual se establece la base legal para que el Ecuador acoja la normativa internacional en el sector público no financiero, el articulado que sustenta lo manifestado es el siguiente:

Art.70.- Segundo inciso: "Todas las entidades, instituciones y organismos comprendidos en los artículos 225,297 y 315 de la Constitución de la República se sujetarán al SINFIP, en los términos previstos en este código, sin perjuicio de la facultad de gestión autónoma de orden administrativo, económico, financiero, presupuestario y organizativo que la Constitución o las leyes establecen para determinadas entidades". (Asamblea Nacional, 2010)

Art. 74.- Deberes y atribuciones del ente rector del SINFIP. - numeral 6 "Dictar las normas, manuales, instructivos, directrices, catálogos, glosarios y otros instrumentos de cumplimiento obligatorio por parte de las entidades del sector público para el diseño, implantación y funcionamiento del SINFIP y sus componentes," (Asamblea Nacional, 2010)

Art. 152.- "Obligaciones de los servidores de las entidades. - Las máximas autoridades de cada entidad u organismo público, serán los responsables de velar por el debido funcionamiento del componente de contabilidad gubernamental y los servidores de las unidades financieras, de observar la normativa contable". (Asamblea Nacional, 2010)

Art. 159.- "El ente rector de las finanzas públicas tiene la facultad privativa para expedir, actualizar y difundir los principios, normas técnicas, manuales, procedimientos, instructivos y más disposiciones contables, que serán de cumplimiento obligatorio por parte de las entidades y organismos del Sector Público". (Asamblea Nacional, 2010)

El Reglamento General del Código Orgánico de Planificación y Finanzas Públicas, emitido el 17 de noviembre de 2014, en lo pertinente a la implementación de normas indica:

Art. 164.- Normativa contable aplicable. - "En la formulación de la normativa contable gubernamental el Ministerio de Finanzas considerará las Normas Internacionales de Contabilidad para el sector público y será de cumplimiento obligatorio para las entidades del Presupuesto General del Estado y gobiernos autónomos descentralizados. Para las empresas, banca pública y seguridad social deberán considerarse obligatoriamente los esquemas definidos para la consolidación de cuentas que emita el Ministerio de Finanzas". (Asamblea Nacional, 2014)

En este contexto, de acuerdo a la programación del Proyecto de Convergencia a NICSP, se señala en la tabla 2 el marco regulatorio y de las actividades de implementación de esta nueva herramienta emitido por el Ministerio de Economía y Finanzas 


\begin{tabular}{|c|c|c|}
\hline Fecha & $\begin{array}{l}\text { Acuerdo Emitidos por el } \\
\text { Ministerio de Economía y } \\
\text { Finanzas }\end{array}$ & Disposición \\
\hline 08 de abril de 2019 & $\begin{array}{l}\text { Instructivo para la convergencia } \\
\text { de la Normativa de Contabilidad } \\
\text { Gubernamental a NICSP }\end{array}$ & $\begin{array}{l}\text { Emite el instructivo para la convergencia de la } \\
\text { Normativa de Contabilidad Gubernamental a } \\
\text { NICSP, contempla las condiciones que deben } \\
\text { tener las cuentas de los estados financieros } \\
\text { para que el } 1 \text { de enero de } 2020 \text { se inicie con las } \\
\text { normas internacionales }\end{array}$ \\
\hline 06 de febrero 2019 & 0015 & $\begin{array}{l}\text { La sustitución del año de elaboración de estados } \\
\text { financieros }\end{array}$ \\
\hline 17 de febrero 2019 & 0017 & $\begin{array}{l}\text { Actualización del Catálogo General de Cuentas } \\
\text { Contables del Sector Público No Financiero, que } \\
\text { será de uso obligatorio para todas las entidades } \\
\text { del Sector Público No Financiero }\end{array}$ \\
\hline 17 de diciembre 2018 & 0008 & $\begin{array}{l}\text { Depuración de Saldos Contables / Cuentas por } \\
\text { Pagar Años Anteriores }\end{array}$ \\
\hline 14 de marzo 2018 & 0003 & $\begin{array}{l}\text { Depuración saldos contables cuentas de } \\
\text { inversión, Reconocimiento, acumulación de } \\
\text { costos de inversión y aplicación de gastos de } \\
\text { gestión }\end{array}$ \\
\hline 08 de febrero 2018 & $00-4$ & $\begin{array}{l}\text { Actualizar el catálogo general de Cuentas } \\
\text { Contables que será de uso obligatorio para todas } \\
\text { las entidades del Sector Público No Financiero }\end{array}$ \\
\hline 10 de noviembre 2017 & 0004 & Depuración de cuentas contables Institucionales \\
\hline 20 de abril 2016 & 0067 & $\begin{array}{l}\text { Expedir los Principios y Normas Técnicas de } \\
\text { Contabilidad gubernamental actualizados }\end{array}$ \\
\hline 06 de abril 2016 & 0077 & $\begin{array}{l}\text { Se incorpora las Políticas, Marco Conceptual } \\
\text { y Normas Técnicas de la Contabilidad } \\
\text { Gubernamental }\end{array}$ \\
\hline
\end{tabular}

Fuente: (Ministerio de Economía y Finanzas, 2019)

\section{El grado de avance de convergencia} de la contabilidad gubernamental en la provincia del Cañar con las NICSP, se relaciona con lineamientos emitidos por el ente rector del país. A continuación, se exponen la matriz de frecuencia relativa de los resultados de la encuesta aplicada a los funcionarios de los gobiernos autónomos descentralizados de la provincia del Cañar (tabla 3) 
Contabilidad gubernamental y normas internacionales de contabilidad para el sector público ecuatoriano

Reyes Reinoso, Johanna Rosalí; Reyes Cárdenas, Narciza y Cárdenas Muñoz, Jorge_

\section{Tabla 3}

Matriz de frecuencia relativa de los resultados de la encuesta

\begin{tabular}{ccc}
\hline Número de pregunta & Si & No \\
\hline 1 & $60 \%$ & $40 \%$ \\
2 & $80 \%$ & $20 \%$ \\
3 & $40 \%$ & $60 \%$ \\
4 & $80 \%$ & $20 \%$ \\
5 & $40 \%$ & $60 \%$ \\
6 & $20 \%$ & $80 \%$ \\
7 & $80 \%$ & $20 \%$ \\
8 & $20 \%$ & $80 \%$ \\
9 & $100 \%$ & $0 \%$ \\
10 & $60 \%$ & $40 \%$ \\
11 & $40 \%$ & $60 \%$ \\
12 & $80 \%$ & $20 \%$ \\
13 & $60 \%$ & $40 \%$ \\
14 & $40 \%$ & $60 \%$ \\
15 & $80 \%$ & $20 \%$ \\
16 & $0 \%$ & $100 \%$ \\
17 & $40 \%$ & $60 \%$ \\
& 54,12 & 45,88 \\
\hline Promedio & & \\
\hline
\end{tabular}

Fuente: Elaboración propia

El grado de avance en la ejecución de la convergencia de la contabilidad gubernamental en la provincia del Cañar con las NICSP se puede observar en el gráfico 2. 


\section{Gráfico 2 \\ Nivel de cumplimiento a los requerimientos para la convergencia a las NICSP de la Provincia del Cañar}

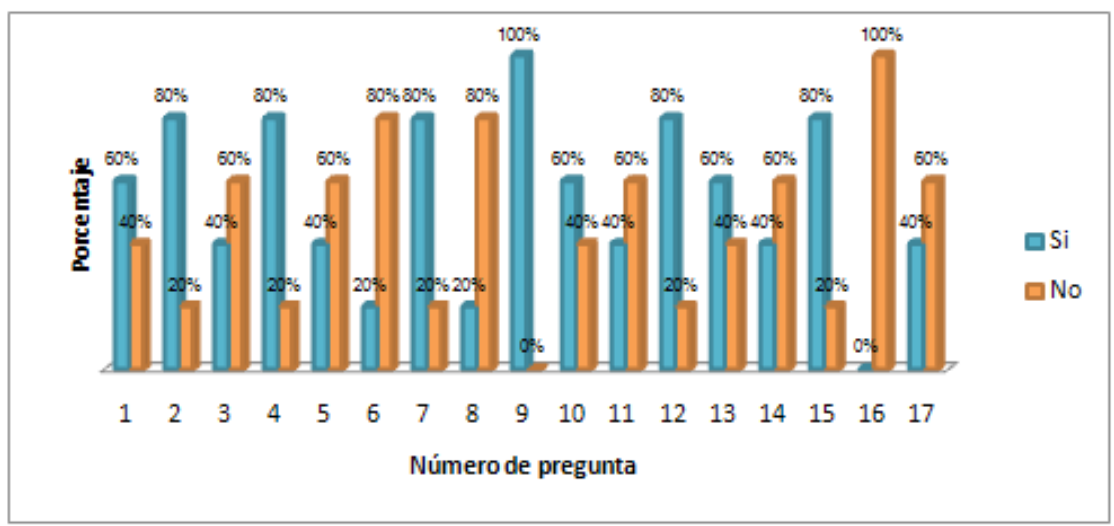

Fuente: Elaboración propia

Respecto al conocimiento emitido por el ente rector para la convergencia de la Normativa de la Contabilidad Gubernamental a NICSP (item1), el $60 \%$ de los encuestados de los Gobiernos Autónomos Descentralizados Municipales manifestó que conoce el instructivo, mientras que el $40 \%$ indicó no haber revisado dicho instructivo.

Sobre el cumplimiento de la normativa del subgrupo de cuentas 112 (ítem 2) el 80\% de los gobiernos autónomos tiene previsto amortizar o recaudar los anticipos no contractuales al finalizar el ejercicio 2019. El $20 \%$ considero no cumplir con la normativa para final del ejercicio ya que existen problemas para legalizar algunos anticipos.

En cuanto al cumplimiento con la normativa del subgrupo de cuentas 113 y 124 (ítem 3) el 40\% de los encuestados manifestó que su administración cumplirá para la fecha prevista la recaudación y/o liquidación de las cuentas por cobrar. En tanto, el $60 \%$ expresó no cumplir con dicha meta, bien porque necesitan acciones interdepartamentales para cumplir con lo requerido o se necesita primero sanear el catastro de su jurisdicción.

En relación con el cumplimiento de la normativa del subgrupo de cuentas 121,122 y 123 (ítem 4), el $80 \%$ considera que sus gobiernos tendrán actualizados los saldos de inversiones financieras con valor razonable al finalizar el ejercicio contable 2019. Mientras que el $20 \%$ de los gobiernos municipales no manejan estos saldos.

En cuanto al cumplimiento de tener en el balance al final del periodo contable 2019, las cuentas de activos diferidos amortizado a valor razonable (ítem 5), el $60 \%$ de los gobiernos no tienen este tipo de cuentas, mientras que el $40 \%$ restante que sí las tiene, piensa que cumplirá con este requerimiento. 
Reyes Reinoso, Johanna Rosalí; Reyes Cárdenas, Narciza y Cárdenas Muñoz, Jorge_

En concordancia con el cumplimiento de la normativa del subgrupo de cuentas 126 (ítem 6), el $80 \%$ de los gobiernos autónomos municipales manifestó no poseer este grupo de cuentas relacionadas con cuentas de dudosas recuperación, en tanto el $20 \%$ restante que sí las posee, piensa que cumplirá con la norma.

Con relación al lineamiento sobre los procesos de análisis y prescripción de cuentas 212, 213, 224 y 226 (ítem 7 ), el $80 \%$ de las distintas jurisdicciones consultadas opinó que cumplirá con dicho lineamiento, mientras que el $20 \%$ indico desconocer dicha normativa.

Respecto al cumplimiento con la normativa del subgrupo de cuentas 225 (ítem 8), el $80 \%$ de los gobiernos municipales no tiene este tipo de cuentas referidas a créditos diferidos, mientras que el $20 \%$ de los gobiernos que si la poseen manifestaron cumplir con esta obligación al finalizar el ejercicio 2019.

El $100 \%$ de los gobiernos autónomos cumplirá con la normativa del subgrupo de cuentas 221,222 y 223 (ítem 9) vinculado a la actualización al final del ejercicio 2019 de las cuentas de títulos, valores y empréstito.

En cuanto a la conciliación de los saldos de bienes e inventario al final del periodo contable 2019 (ítem 10), el 60\% de los gobiernos municipales considera que podrá cumplir con esta exigencia al finalizar el ejercicio 2019, mientras que el $40 \%$ opina que no podrá cumplir por falta de consolidación entre contabilidad y bodega.

En relación al lineamiento de la normativa sobre la revalorización de los saldos de bienes (ítem 11), el 60\% de los gobiernos autónomos opinaron que no podrán cumplir ya que los bienes no están revalorizados a la fecha o los saldos no están consolidados entre contabilidad y bodega. Mientras que el $40 \%$ de los gobiernos municipales restante consideraron que podrán cumplir con este lineamiento.

Respecto al cumplimiento con la normativa sobre la documentación que avale el dominio de propiedad de los bienes (ítem 12) el $80 \%$ de las jurisdicciones consultadas opinaron poder cumplirlas, el $20 \%$ restante opinó que no podrá cumplirla ya que los inmuebles no poseen escritura que den dominio de propiedad a la entidad.

En cuanto al procedimiento sobre la depuración de la información financiera (ítem 13), el $60 \%$ de los gobiernos municipales consultados prevén tener la información financiera depurada al finalizar el ejercicio. Mientras el $40 \%$ manifestó no cumplir con la normativa, bien porque es necesario generar los ajustes y recabar la documentación pertinentes que los sustentes, bien porque no cuentan con saldos conciliado en los bancos desde el año 2015 o porque los anticipos deben ser saneados.

Sobre el cumplimiento del lineamiento sobre saldos de las cuentas inmuebles e infraestructura para ser trasladado al subgrupo de cuentas 143 (ítem 14), el 60\% de los gobiernos municipales no podrá cumplir con este lineamiento, ya que, de acuerdo a lo manifestado por los encuestados, no tiene que realizar ajustes o no cuentan con información requerida para este proceso. En tanto que el $40 \%$ de los gobiernos municipales aspira a cumplirlo al finalizar el periodo 2019.

Respecto al cumplimiento del lineamiento referido a los saldos de inventario conciliados a través del método promedio ponderado que reflejen el valor real (ítem 15), el $80 \%$ de los gobiernos municipales esperan 
cumplirlos al finalizar el periodo 2019, el $20 \%$ restante señalaron no contar con kárdex con método promedio ponderado.

El cuanto a la posesión de bienes intangibles (ítem 16), el $100 \%$ de los gobiernos municipales declaró no poseer bienes intangibles.

En relación al lineamiento de prevención de actas de entrega e incorporación en las distintas cuentas contables de los proyectos de construcción de bienes inmuebles, infraestructura y/o bienes nacionales tangibles o intangibles (ítem17), el 60\% de los gobiernos municipales piensa que no podrán cumplirlos ya que, o bien falta documentación y trámites internos, se requieren de actas para ajustar o están recabando los documentos para realizar el ajuste.

Igualmente se identificaron problemas en los gobiernos autónomos de la provincia del Cañar que podrían retrasar la convergencia, tal como se muestra en el gráfico 3 , los mismos responden a la falta de coordinación interdepartamental que ocasiona que el departamento contable no cuente con los documentos necesarios que sustente el registro de los hechos económicos como lo requiere la norma respectiva y por lo tanto los saldos no sean razonables y no estén sustentados conforme a la normativa internacional

\section{Gráfico 3}

Principales problemas identificados para el cumplimiento de la convergencia a las NICSP de la Provincia del Cañar

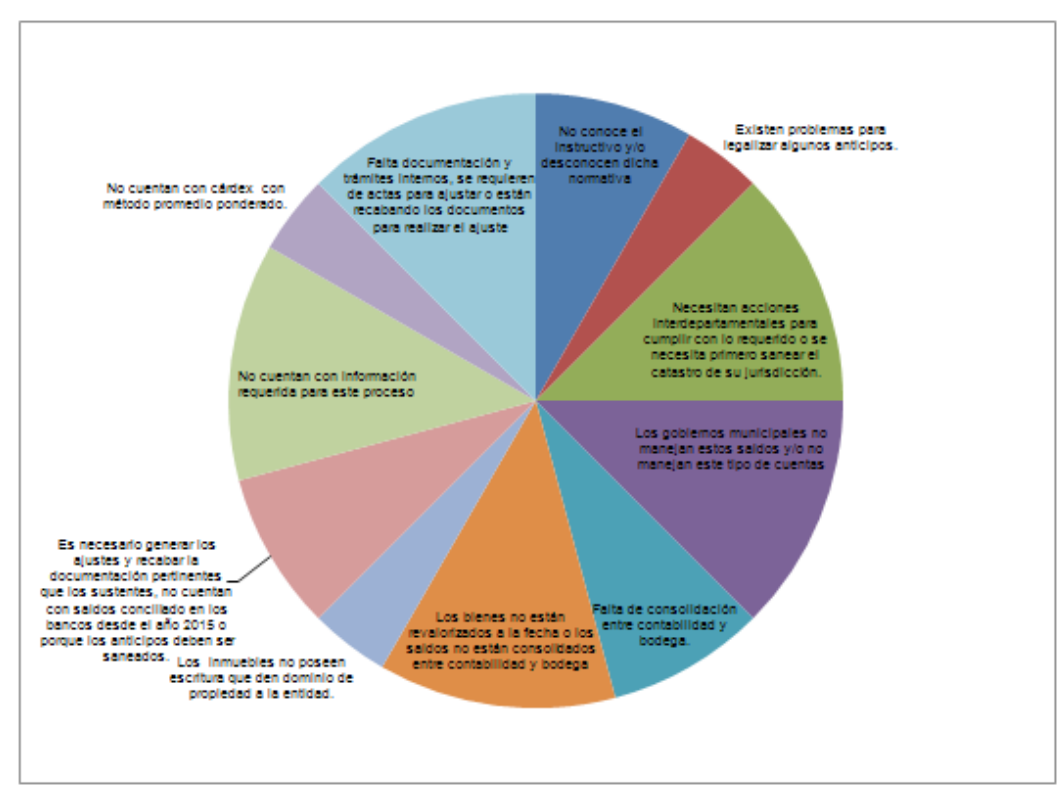

Fuente: Elaboración propia 
Reyes Reinoso, Johanna Rosalí; Reyes Cárdenas, Narciza y Cárdenas Muñoz, Jorge_

Adicionalmente, se identificó la carencia de documentos que sustenten el dominio de bienes de la entidad, lo que agrava la carencia de la revalorización de la cuenta propiedad, planta y equipo de aquellos que están con vida residual. Se identificó además la falta de conciliación de los bienes y la manera adecuada del control de inventario, es decir con la valoración a través del método promedio ponderado establecido en la norma técnica de contabilidad gubernamental.

En este sentido, en promedio el $46 \%$ de los requerimientos para converger a la normativa internacional de los gobiernos municipales de la provincia Cañar no están adecuados para que se pueda cumplir a la fecha prevista por el ente rector, ya que los aspectos que hay que corregir les va a tomar un tiempo superior a los casi cinco meses que tienen para ello.

Al ser la contabilidad gubernamental, una herramienta que tiene como uno de sus fines el control los recursos públicos, y por lo tanto evitar el desvío de los mismos en práctica reñidas con la ética, es necesario que la misma esté realizada conforme lo prevén las normas, situación que se ve no está pasando en las jurisdicciones estudiadas y por lo tanto el control es deficiente.

Por lo antes expuesto, se hace necesario que las universidades del país, se aboquen al estudio y acompañamiento en este proceso de transformación de la contabilidad gubernamental, para minimizar los efectos negativos en este proceso de transición. El gráfico 2 muestra el cumplimiento de los lineamientos para la convergencia a las NICSP de la Provincia del Cañar.

\section{Conclusiones}

Ecuador busca armonizar sus normas de Contabilidad Gubernamental con la finalidad de hacer más competitiva su información y mejorar su gestión administrativa financiera.

La adaptación a las NICSP de la contabilidad pública, Ecuador intenta promover una transformación en la administración financiera pública, en este sentido, afectará las normas y principios contables establecidos en todo el subsistema financiero de los entes públicos.

Los resultados muestran que las entidades municipales estudiadas, tienen deficiencias para cumplir con los lineamientos de convergencia para la adopción de estas normas, pues se identificaron problemas que pudieran retrasar su implementación.

Es necesario que el personal de las diferentes áreas financieras y administrativas de las entidades municipales responsables de la aplicación de las NICSP, se encuentre capacitado y promuevan la participación activa sobre las normas a ser aplicadas lo que conllevará a una retroalimentación continua entre las diferentes coordinaciones interdepartamental.

Los temas complejos y novedosos que comprenden estas normas provocan incertidumbre y duda en los distintos actores de la contabilidad gubernamental en la Provincia del Cañar. Por lo que se hace necesario el diseño de un plan integrado, entre los entes municipales descentralizados, el ente rector y las universidades para cumplir con los lapsos requeridos. 


\section{Referencias bibliográficas}

Ablan Bortone, Nayibe Carolina (2013), Las Normas Internacionales de Contabilidad para el Sector Público (NICSP): una revisión de los aspectos clave a considerar y de la situación en Venezuela, Visión Gerencial, número 2, Venezuela, Universidad de los Andes, pp 221-240.

Alvarado Mareina, José (1995), Contabilidad gubernamental, Primera edición, México, Acuario Editora.

Asamblea Nacional (2010), Código Orgánico de Planificación y Finanzas Públicas, Registro oficial 306, Quito, Ecuador.

Asamblea Nacional (2014), Reglamento al Código Orgánico de Planificación y Finanzas Públicas.: Registro Oficial 383, Quito, Ecuador

Banco Interamericado de Desarrollo BID (2017), Estado de adopción de NICSP en los países de Latinoamérica y el Caribe, Washington D.C, Firma auditora independiente EY.

Casal, Rosa (2007), La ciencia contable, su historia, filosofía, evolución y su producto, Actualidad Contable FACES, volumen 10, número 15, Venezuela, Universidad de los Andes, pp 19-28.

Fuertes, lluminada (2007), Un Análisis del grado de comparabilidad de la información contable pública basado en la rigidez del marco regulador del IFAC, Presupuesto y Gasto Público, www.ief.es/documentos/.../publicaciones/...publico/47_Fuertes. pdf. [Consulta: Agosto 10,2019$]$.

Gianchino, Carmen (2011), Implementación de normas internacionales de contabilidad para el sector público - NICSP Debilidades y Fortalezas, San Juan, Puerto Rico, Traba- jo Técnico de la XXIX Conferencia Interamericana de Contabilidad.

Lara, Marco, Toledo, Indra, \& Gómez, Luis (2012), La rendición de cuentas por el gobierno electrónico. Caso entidades federativas de México. Revista Cuadernos de Contabilidad, volumen 13 , número 33 , Colombia, pp 463-478.

López de Sá, Antonio (2002), Orígenes y evolución del conocimiento contable. Colombia, Editorial Panamericana.

Mejías, Juan (2011), La crisis de la prudencia en la convergencia contable internacional, Revista Internacional Legis de Contabilidad \& Auditoría, número 48, Colombia, pp 135-182.

Ministerio de Economía y Finanzas (17 de julio de 2019), Normativa de Contabilidad Gubernamental, Obtenido de Normativa de Contabilidad Gubernamental: https://www.finanzas.gob.ec/normativa-de-contabilidad-gubernamental/

Montesino, Vicente (2003), Panorama actual de la contabilidad pública: Análisis de la situación española dentro de su entorno internacional, CIRIEC-España, Revista de Economía Pública, Social y Cooperativa, número 15, España, pp 159-185.

Presidencia de la República (2014), Reglamento General del Código Orgánico de Planificación y Finanzas Públicas, Quito, Pichincha.

Ramos, Cristian (2012), Importancia del estudio de la evolución de la contabilidad desde una perspectiva científica, Cartagena, Universidad de Cartagena.

Rodríguez, Manuel, Navarro, Andrés, \& Alcaraz, Francisco (2014), La mejora de la rendición financiera de cuentas para modernizar el sector público de 
Contabilidad gubernamental y normas internacionales de contabilidad para el sector público ecuatoriano

Reyes Reinoso, Johanna Rosalí; Reyes Cárdenas, Narciza y Cárdenas Muñoz, Jorge_

países emergentes de América Latina, Revista del CLAD Reforma y Democracia, número 59, Venezuela, pp 83-118.

Zerpa, Sadcidi , Rosales, Maritza \& Morales, Odalis (2017), Contabilidad en el sector público y empresarial en Venezuela, Gestión y Gerencia, volumen 12 , número 2 , Universidad Centroccidental Lisandro Alvarado, Venezuela, pp 1-19.

- Esta obra está bajo una licencia de Creative Commons Reconocimiento-NoComercialCompartirlgual 3.0 Unported.

http://creativecommons.org/licenses/by-nc-sa/3.0/deed.es_ES 